original paper

doi: https://doi.org/10.5114/pq.2023.112744

\title{
Effects of circuit exercise training on body image, cardiorespiratory indices, and body composition of obese undergraduates in a Nigerian University
}

Jeneviv John ${ }^{1}$, Ujunwa Nnaji ${ }^{1}$, Obinna Okezue ${ }^{1}$, Ugochukwu Nnadozie ${ }^{2}$, Davidson John ${ }^{3,4}$, Antoninus Ezeukwu ${ }^{1}$, Ukamaka Mgbeojedo ${ }^{1}$, Ifeanyi Enyanwuma ${ }^{2}$

${ }^{1}$ Department of Medical Rehabilitation, Faculty of Health Sciences and Technology, University of Nigeria

${ }^{2}$ Division of plastic surgery, Department of Surgery, Alex Ekwueme Federal University Teaching Hospital, Abakaliki, Nigeria

${ }^{3}$ Department of Physiotherapy, Alex Ekwueme Federal University Teaching Hospital, Abakaliki, Nigeria

${ }^{4}$ Department of Physiotherapy, Evangel University, Ebonyi State, Nigeria

Correspondence address: John Davidson Okwudili, Department of Physiotherapy, Alex Ekwueme Federal University Teaching Hospital, PMB 102 Abakaliki, Nigeria,

e-mail:davidsonokwudili@yahoo.com

\section{ABSTRACT}

Introduction. Obesity is a disease that adversely affects quality of life, mortality and morbidity and it is associated with a sedentary lifestyle. This study evaluated the effect of circuit-exercise training on the cardiorespiratory indices, body image, and body composition in obese undergraduate students.

Methods. Ninety obese undergraduates (69 females and 21 Males) were assigned to either a circuitexercise training group/study or a non-exercise/control group. The exercise program comprised of 40 minutes of circuit-training (resistance and aerobic) sessions, performed 3 times per week for 6 weeks. Participants rotated between stations with 20 seconds rest in between; as well as warm up and cool down periods. Moderate exercise-intensity was performed at 50\%-70\% of the agepredicted heart rate reserve. Pre (baseline) and post (at 6 weeks) values of cardiovascular parameters, body image, and body composition parameters were evaluated. Demographic data were summarized with descriptive statistics while significant differences within and between groups were determined using paired and independent sample $t$-tests, respectively. Statistical significance was accepted for all tests at a value of $p<0.05$.

Results. Findings from the results showed no significant difference in the mean body image, body mass index, and waist-hip ratio between the groups $(p>0.05)$. A significant difference was observed between the groups $(p<0.05)$ in the mean aerobic capacity, systolic and diastolic blood pressure, heart rate, percentage body fat, and muscle mass.

Conclusion. A 6-week circuit training had a favorable but not significant effect on body image. Aerobic capacity, cardiovascular parameters, and some body composition parameters had significant changes.

Key words: circuit training, obesity, cardiorespiratory fitness, body composition, body image

\section{Introduction}

Obesity is a medical condition depicted by the accumulation of excess body fat stored to an extent that it may negatively affect health [1]. Studies have reported a steady rise in the obese population and have attributed this to a decline in physical activity and excessive intake of food [2]. Lack of exercise/sedentary lifestyle is considered one of the most important health concerns associated with modern society [3]. This is implicated in the development of many chronic illnesses 
and disorders and increased risk factors for the rise in cardiovascular disease [4]. The emergence of illnesses can greatly be reduced through regular exercise. Hence exercise performance is known to be linked to body weight loss as well as reduction in all-cause mortality and chronic diseases such as cardiovascular disease and diabetes, leading to an increased life expectancy and mitigating the negative health impact of obesity [5]. In addition, it has a positive impact on the psychological wellbeing and body image of an individual [6].

More so, body image (BI) is an individual's psychological and mental experience of the appearance of his/her body [7]. Beliefs regarding some sort of perfect body image, the tendency for people to compare themselves to others, and a sense of insecurity are some of the major psychological correlates of body image satisfaction. There is strong evidence of a negative perception of body image by most women and girls [8]. Many are not satisfied with their body size and weight since slimness is seen as the fascinating standard and beauty model, especially for young women [9]. Body image may be seen as the fulcrum of the mutual influences of the physique, dietary and exercise lifestyle on each other. It is a determining factor of self-esteem which includes perceptual, affective, and mental components, which are based partly on the composition of an objective anthropometric representation [9.]

Furthermore, body image literature has most commonly used body mass index (BMI) as an anthropometric measure. While it is inversely associated with body satisfaction [10], BMI has been criticized because it does not discriminate/differentiate between fat mass and fat-free mass in the body. Body composition is the proportion of fat mass and fat-free mass in the body. Body fat is a measurement of body composition estimating the amount of body weight that is fat while fat-free mass is the percentage of body weight that is not fat. Body fat percentage is the total weight of body fat divided by body weight and it consists of storage and essential body fat [11]. Excessive body fat or obesity is linked to increased metabolic risk, and its measurement is vital in implementing preventive and curative health strategies [11]. Generally, people are considered obese when their BMI (body weight divided by the square of the height) is $\geq 30 \mathrm{~kg} / \mathrm{m}^{2}$. The unceasing epidemic of obesity in adults and children has brought to fore the implication of body fat for short term and long term health. Consequently, obesity is a preventable but a leading cause of death worldwide; with increasing incidence in children and adults [1]. American medical association classified obesity as a disease in 2013 [12].

However, the long term fitness and health benefits of exercise performance include the reduction of risk factors of most chronic diseases as well as the improvement of muscle strength and endurance, flexibility, body composition, and cardiorespiratory functions [13]. In the past, many guidelines have focused on one mode of resistance training or aerobic training to facilitate weight loss or weight maintenance, but recent facts are emerging that circuit exercise training can also be effective for reducing fat mass [14].

Circuit exercise training (CET) is a type of combined-training which includes both multi-joint resistance training and aerobic exercise. Some researchers have suggested that a CET composed of endurance and resistance training may be preferred to one focused only on a single mode of exercise [14]. The training session includes several stations where different exercises are performed for a set time and after the appointed time the station is changed as quickly as possible. Both resistance training and aerobic training have been prescribed for obese individuals, because they promote heart rate and blood pressure regulation, body composition, biochemical markers, as well as muscle strength and aerobic capacity $[15,16]$. However, except for the aforementioned, another goal may include improving body image, which is associated more with psychological well-being than with physiological well-being. With these findings, indulging in exercise has been proved to be vital for the prevention and treatment of physiological and psychological problems in the obese population. Hence, this study is focused on ascertaining the effects of 6 weeks of circuit exercise training on body image, cardiorespiratory parameters, and body composition of obese undergraduate students.

\section{Subjects and methods}

Research design

A pre and post controlled randomized study. 
The sample size was calculated as 30 using a power analysis that showed that to get a small effect size $(\mathrm{d}=0.95)$ at a power of 0.8 and 0.05 level of significance, a minimum sample size of 30 subjects is needed. Therefore, a minimum of 30 subjects was needed for this study.

\section{Subject selection}

The database of the students was accessed from the students' affairs department of the campus where the study was carried out. Text messages and e-mail were sent to students informing them of the study and requesting them to declare an interest. One hundred and seventy students declared an interest and were invited to the study. However, only one hundred and twenty students eventually reported for the study. One hundred and one met the selection criteria for participation in the study. Inclusion criteria were healthy obese undergraduate who are willing and available to participate in the study, BMI $\geq 30 \mathrm{~kg} / \mathrm{m} 2$, age 18years, and above. Exclusion criteria were students with BMI < $30 \mathrm{~kg} / \mathrm{m} 2$, students who are currently on an exercise program, students who are on dietary restriction, presence of contraindication to exercise testing and training, students with significant cognitive impairment or inability to follow instructions, students who are pregnant. A total of one hundred and one subjects were recruited for the study (50 subjects in the control group and 51 subjects in the study group). Fishbowl simple random sampling method was used to randomize subjects into a study group (51 subjects) or a control group (50 subjects). Numbers were written on cards and draws were made.

The subjects were made up of 69 females and 21 males. However, a total of eleven subjects did not complete the course of the study. Five dropped out of the control group while four dropped out of the experimental group while the remaining two did not attain the eligibility $70 \%$ attendance during the exercise training. All the subjects were sedentary and were assigned either to the study or control group randomly.

\section{Instruments}

An adapted body image questionnaire from the cosmetic procedures screening questionnaire (COPS) was used to assess the body image of the subjects. This questionnaire is a nine-item selfreport measure of body dysmorphic disorder (BDD) symptoms. It generates a total score ranging from 0 to 72 . It measures the severity of BDD symptoms and identifies people with BDD. A positive and negative affect score was used in assessing the impact of the CET on the body image of the subjects. The COPS has good internal consistency $(\alpha=0.86-0.91)$, good test-retest reliability, evidence of single-factor structure, and moderate to good corrected item-total correlations $(\mathrm{r}=0.41$ 0.86) [17].

OMRON Body composition monitor (BF511, OMRON HEALTHCARE Co. Ltd, Kyoto Japan) was used to measure body composition. This device is made up of the main unit which has a pair of electrodes for the foot and a display unit which has a pair of electrodes for the handgrip. The main unit is connected to the display unit via a cord. The device uses the bioelectrical impedance method to measure the body fat $(\mathrm{BF})$ percentage. Age, height, gender, and weight of the subjects are inputed into the device which were taken into consideration to generate the following body composition parameters: total BF (\%), visceral fat (VF) (\%), BMI, body weight (BW), muscle mass (MM), and resting metabolic rate. This device has been reported to be a valid assessor of body composition parameters [18].

Tape rule (HD2020, Shanghai Kearing Stationery Co., Ltd., Shanghai, China): The waist circumference (WC) in centimeters was measured with a non-elastic tape measure which ranges from $0-150 \mathrm{~cm}$ was used to measure.

Stadiometer: A locally constructed stadiometer made of wood and calibrated in centimeters $(\mathrm{cm})$ was used to measure the height of the subjects.

Digital exercise Metronome (JM-06, Superior Medical Equipment Inc, Wilmington, United States of America): A digital metronome provided a rhythmic cadence which produced a steady pulse (or beat) that helped maintain an established tempo during exercise sessions. This was used to bring about uniformity in the movement of subjects during exercise sessions. 
Digital sphygmomanometer (HEM7120, Omron Healthcare Co. Ltd, Kyoto, Japan): This was used for checking the systolic and diastolic blood pressure.

Kadio Stopwatch (KD-1063, Takshun electronic Co. Ltd, Fujian, China): This was used to track the duration of the exercise sessions.

Exercise mats (Dynamos $18 \mathrm{~cm} \times 60 \mathrm{~cm} \times 15 \mathrm{~cm}$, Decathion, UK): Used for resistance exercises during the circuit training sessions that require lying down e.g. crunches.

\section{Procedure for data collection}

The subjects were converged at the University indoor sports gym. They were intimated on the aims, procedures, and task requirements for the data collection. They were informed of their supposed roles and hopeful benefits to gain, while also assuring them of confidentiality during the study.

Subjects in both groups were placed on a diet plan by a dietician. They were encouraged to eat healthy, properly cooked, balanced diets and to eliminate the intake of "junks", which is customary of undergraduates. They were instructed not to skip nor eat in-between meals. They were given specific time durations to take each meal of the day and asked not to take more than one proportionate serving per meal.

\section{Cardiorespiratory indices}

The vital signs were taken at the beginning and end of each exercise session. The pulse rate was measured in beats per minute (bpm), for a full one minute using a stopwatch. A sphygmomanometer was used together with a stethoscope for measuring the blood pressure in millimeters per mercury $(\mathrm{mmHg})$. Cardiorespiratory fitness or maximum oxygen consumption $\left(\mathrm{VO}_{2} \mathrm{max}\right)$ was predicted using the heart rate ratio method [19]. The formula is as follows: $\mathrm{VO}_{2} \max$ $\left(\mathrm{mL} \cdot \mathrm{min}^{-1} \cdot \mathrm{kg}^{-1}\right)=15.0 \times($ HRmax $/$ HRrest $)$.

HRmax $=(220-$ Age $)$, HRrest $=$ Heartbeats $/ 1$ minute. All values were taken at baseline and at 6 weeks.

\section{Body composition}

The height of each subject was measured by instructing them to stand in an upright position in front of the stadiometer without wearing any footwear. The readings were taken off from the stadiometer using a ruler placed horizontally on the vertex of the subject's head. The readings were read off to the nearest 0.1 centimeters [20].

OMRON BF511 was used to measure the weight $(\mathrm{kg})$, as the subject stepped on the main unit barefooted in an upright position with the feet placed on the foot electrodes and the weight evenly distributed.

OMRON BF511 measured the following parameters: BMI $\left(\mathrm{kg} / \mathrm{m}^{2}\right)$, Visceral fat $(\mathrm{VF})$ percentage, body fat (BF) percentage and skeletal muscle mass (MM). Measurements of age, weight, height, and gender were integrated to generate these parameters using the bio-electric impedance method.

Waist and hip circumference

Subjects stood in an upright position and measurement of the waist circumference was taken between the lower costal border and the narrowest point of the iliac crest at the end of normal expiration. The subject's arm was in abduction so as to ensure that the tape was passed around the abdomen with ease [20]. Measurement of the hip circumference was taken with the patient in standing position with the arms folded across the chest, the feet together and the gluteal muscles relaxed [20]. Measurement was taken at the level of the greatest posterior protuberance of the buttocks. The waist-hip ratio was calculated pre (baseline) and post (at 6 weeks).

An adapted body image questionnaire was handed over to all the participants to either fill on-site or take home as they deem fit. The questionnaire was issued at baseline and at 6 weeks. 
Prior to the initiation of exercise training, subjects were acquainted with techniques required for the proper performance of the exercise. The subjects were trained 3 times a week (with at least 1 day of rest between sessions) for six weeks at 60 minutes per session, under the supervision of a physiotherapist. A physician who is also part of the team was always on standby in case of any emergency during the exercise session. The warm-up and cool-down periods were integrated into each exercise session involving 10 minutes of low-intensity and light stretching activities. The CET included a mixture of 10 types of both resistance and aerobic exercise. The resistance exercises included squats, crunches, push-up, superman exercise, and side lunge exercise while the aerobic exercises included light jumping, running on the spot, jumping jack, foot-stamping, walking in place. Each exercise in the training program was performed for 60 seconds with a resting time of 20 seconds between stations and 3 minutes between sets. Two to four sets of the exercise were performed per session. The rest period and the number of repetitions were adjusted as the week progressed and the fitness level of the subjects increased. Table 1 shows the progression of the CET. The exercise was performed at a moderate intensity level of 50\%-70\% of the predicted maximum heart rate. The target heart was calculated by the Karvonen formula using the heart rate reserve (HRR) [21].

HRR $=(220-$ Age $)-$ Resting heart rate (HRrest).

Target Heart rate $=($ HRR $x \%$ intensity $)+$ HRrest

Table 1. Circuit exercise training programme progression

\begin{tabular}{|l|l|l|l|}
\hline Parameter & & Events & Intensity \\
\hline Warm-up & Dynamic stretching & & $40 \%-50 \%$ HRmax \\
\hline \multirow{2}{*}{$\begin{array}{c}\text { CIRCUIT } \\
\text { TRAINING }\end{array}$} & Resistance exercise & $\begin{array}{l}\text { Push-up, squat, crunches, } \\
\text { lunge and superman exercise }\end{array}$ & $\begin{array}{l}\text { Weeks 1-3, } \\
50 \%-60 \% \text { HRmax }\end{array}$ \\
\cline { 2 - 4 } & Aerobic exercise & $\begin{array}{l}\text { A light jumping, running on } \\
\text { the spot, foot stamping, steps, } \\
\text { jumping jack }\end{array}$ & $\begin{array}{l}\text { Weeks 3-6, 60\%- } \\
70 \% \text { HRmax }\end{array}$ \\
\hline Cool-down & Static stretching & & 40\%-50\% HRmax \\
\hline
\end{tabular}

HRmax $=$ maximum heart rate

Data analysis

Data were summarized using descriptive statistics of mean, standard deviation, percentages and frequency. The normality of data distribution was tested and confirmed by Shapiro-Wilks test. The mean differences of variables between the groups were compared using an independent sample $t$ test while the mean differences of variables within the group were tested with the paired t-test. Alpha level was set at $p<0.05$. A statistical package for the social sciences (SPSS version 21) was used to analyze all data.

\section{Ethical approval}

The research related to human use has complied with all the relevant national regulations and institutional policies, has followed the tenets of the Declaration of Helsinki, and has been approved by the University of Nigeria Teaching Hospital (NHREC/05/01/2008B-FWA000024581RB00002323).

\section{Informed consent}

Informed consent has been obtained from the subjects before participation.

\section{Result}

A total of 90 subjects (45 in the control group and 45 in the study group) with the age range of 18 - 29 years completed the study. All measurements were taken pre (baseline) and post (at 6week) and the results were analyzed at $p<0.05$ and a confidence interval of $95 \%$. The socio- 
demographic characteristics of the subjects are summarized in (Table 2) below. There where no significant diference in all the baseline parameters between the groups.

The between-group comparison showed a significant difference in all the cardiorespiratory parameters $(p<0.05)$ post-intervention (Table 3$)$. While within-group comparison (Table 4$)$ in the study group also showed a significant difference in cardiorespiratory parameters $(p=0.01)$. Comparison of body composition between the groups (Table 5) post-intervention showed a significant difference only in MM, WC, and \% body fat $(p<0.05)$

Body Image and all the body composition parameters revealed a significant difference $(p<0.05)$ within the study group (Table 6). While only BI, BMI, \%BF, and $\mathrm{MM}$ showed a significant difference $(p<0.05)$ within the control (Table 6).

Table 2. Socio-demographic statistics of participants

\begin{tabular}{|l|l|c|c|c|c|}
\hline Variable & Category & $\begin{array}{c}\text { Control } \\
(n=45)\end{array}$ & $\begin{array}{c}\text { Study } \\
(n=45)\end{array}$ & $t$-value & $P$-value \\
\hline Age & Years & $21.40 \pm 3.12$ & $21.36 \pm 4.14$ & 0.218 & 0.525 \\
\hline Height & Meters & $1.67 \pm 6.35$ & $1.67 \pm 5.85$ & 0.039 & 0.782 \\
\hline & & Frequency $(\%)$ & Frequency $(\%)$ & Total & \\
\hline Gender & Male & $8(8.9 \%)$ & $11(12.2 \%)$ & $19(21.1 \%)$ & \\
\hline & Female & $37(41.1 \%)$ & $34(37.8 \%)$ & $71(78.9 \%)$ & \\
\hline Ratio & Male:Female & $1: 3$ & $1: 3.6$ & $1: 3.7$ & \\
\hline Total & & $45(50 \%)$ & $44(50 \%)$ & $90(100 \%)$ & \\
\hline
\end{tabular}

Table 3. Comparison of cardiorespiratory parameters between the groups

\begin{tabular}{|l|c|c|c|c|}
\hline Variable & $\begin{array}{c}\text { Study group } \\
(n=45)\end{array}$ & $\begin{array}{c}\text { Control group } \\
(n=45)\end{array}$ & $t$-value & $P$-value \\
\hline Pre & $133.2 \pm 12.13$ & $131.7 \pm 14.66$ & 0.312 & 0.76 \\
\hline SYS $(\mathrm{mm} \mathrm{Hg})$ & $86.53 \pm 10.28$ & $85.8 \pm 12.04$ & 0.179 & 0.86 \\
\hline $\mathrm{DYS}(\mathrm{mm} \mathrm{Hg})$ & $106.83 \pm 12.43$ & $105.73 \pm 12.53$ & -2.423 & 0.16 \\
\hline $\mathrm{HR}(\mathrm{b} / \mathrm{min})$ & $26.11 \pm 3.95$ & $25.27 \pm 2.48$ & 0.64 & 0.53 \\
\hline V0 $2 \mathrm{max}\left(\mathrm{mL} \cdot \mathrm{min}^{-1} \cdot \mathrm{kg}^{-1}\right)$ & $120.6 \pm 7.30$ & $132.00 \pm 15.03$ & -2.64 & $0.01^{*}$ \\
\hline Post & $77.7 \pm 5.50$ & $85.60 \pm 12.33$ & -2.27 & $0.03^{*}$ \\
\hline $\mathrm{SYS}(\mathrm{mm} \mathrm{Hg})$ & $93.0 \pm 6.88$ & $105.00 \pm 3.07$ & -24.37 & $0.03^{*}$ \\
\hline DYS $(\mathrm{mm} \mathrm{Hg})$ & $48.20 \pm 3.00$ & $27.73 \pm 5.82$ & -9.77 & $0.01^{*}$ \\
\hline $\mathrm{HR}(\mathrm{b} / \mathrm{min})$ & $\mathrm{VO}_{2} \mathrm{max}\left(\mathrm{mL} \cdot \mathrm{min}^{-1} \cdot \mathrm{kg}^{-1}\right)$ &
\end{tabular}

SYS - systolic blood pressure; DYS - diastolic blood pressure; HR - heart rate;

$\mathrm{VO}_{2} \mathrm{max}-$ maximum oxygen consumption in one minute; $*$ - statistical significant difference

Table 4. Comparison of cardiorespiratory parameters within the groups

\begin{tabular}{|c|c|c|c|c|}
\hline Variable & Pre & Post & $t$-test & $P$-value \\
\hline \multicolumn{5}{|l|}{ Study group } \\
\hline SYS (mm Hg) & $133.2 \pm 12.13$ & $120.6 \pm 7.30$ & 4.065 & $0.01 *$ \\
\hline DYS (mm Hg) & $86.53 \pm 10.28$ & $77.7 \pm 5.50$ & 3.355 & $0.01 *$ \\
\hline HR (b/min) & $106.83 \pm 12.43$ & $93.0 \pm 6.88$ & 4.163 & $0.01 *$ \\
\hline $\mathrm{VO}_{2} \max \left(\mathrm{mL} \cdot \min ^{-1} \cdot \mathrm{kg}^{-1}\right)$ & $26.11 \pm 3.95$ & $48.20 \pm 3.00$ & 4.260 & $0.01 *$ \\
\hline \multicolumn{5}{|l|}{ Control group } \\
\hline SYS (mm Hg) & $131.7 \pm 14.66$ & $132.0 \pm 15.03$ & -1.00 & 0.33 \\
\hline DYS (mm Hg) & $85.8 \pm 12.04$ & $85.6 \pm 12.33$ & 0.218 & 0.83 \\
\hline $\mathrm{HR}(\mathrm{b} / \mathrm{min})$ & $105.73 \pm 12.53$ & $105.00 \pm 3.07$ & 0.095 & 0.93 \\
\hline $\mathrm{VO}_{2} \max \left(\mathrm{mL} \cdot \min ^{-1} \cdot \mathrm{kg}^{-1}\right)$ & $25.27 \pm 2.48$ & $27.73 \pm 5.82$ & -1.08 & 0.30 \\
\hline
\end{tabular}

SYS - systolic blood pressure; DYS - diastolic blood pressure; HR - heart rate;

$\mathrm{VO}_{2} \mathrm{max}-$ maximum oxygen consumption in one minute; $*$ - statistical significant difference 
Table 5. Between group comparisons of Body image and Body composition parameters

\begin{tabular}{|l|c|c|c|c|}
\hline Variable & $\begin{array}{c}\text { Study group } \\
(n=45)\end{array}$ & $\begin{array}{c}\text { Control group } \\
(n=45)\end{array}$ & $t$-value & $P$-value \\
\hline Pre & $43.53 \pm 8.76$ & $41.3 \pm 9.04$ & 0.677 & 0.50 \\
\hline BI & $34.6 \pm 3.76$ & $33.2 \pm 3.11$ & 1.169 & 0.25 \\
\hline BMI & $42.9 \pm 6.81$ & $46.4 \pm 5.81$ & -1.550 & 0.13 \\
\hline BF $(\%)$ & $27.0 \pm 4.01$ & $24.1 \pm 2.92$ & 2.228 & 0.34 \\
\hline MM $(\%)$ & $100.07 \pm 7.29$ & $99.6 \pm 12.37$ & 0.444 & 0.66 \\
\hline WC $(\mathrm{cm})$ & $119.73 \pm 9.33$ & $118.3 \pm 7.67$ & -0.61 & 0.55 \\
\hline HC $(\mathrm{cm})$ & $0.837 \pm 0.058$ & $0.87 \pm 0.805$ & -1.32 & 0.20 \\
\hline WHR & $34.5 \pm 8.76$ & $38.7 \pm 10.01$ & -1.14 & 0.26 \\
\hline Post & $32.5 \pm 3.34$ & $32.8 \pm 3.02$ & -0.24 & 0.81 \\
\hline BI & $38.4 \pm 4.23$ & $44.8 \pm 5.45$ & -3.60 & $0.01^{*}$ \\
\hline BMI & $29.9 \pm 2.90$ & $25.1 \pm 2.69$ & 4.727 & $0.01^{*}$ \\
\hline BF $(\%)$ & $92.0 \pm 9.99$ & $97.6 \pm 12.42$ & -3.663 & $0.002^{*}$ \\
\hline MM $(\%)$ & $114.2 \pm 8.69$ & $112.3 \pm 7.47$ & 13.92 & $0.001^{*}$ \\
\hline WC $(\mathrm{cm})$ & $0.808 \pm 0.088$ & $0.868 \pm 0.819$ & -1.94 & 0.06 \\
\hline HC $(\mathrm{cm})$ & WHR &
\end{tabular}

$\mathrm{BI}$ - body image; BMI - body mass index; BF - body fat; MM - muscle mass; $\mathrm{W}$ - waist circumference; HC - hip circumference; WHR - waist-hip ratio; * - statistical significant difference

Table 6: Within group comparison of body image and body composition

\begin{tabular}{|c|c|c|c|c|}
\hline Variable & Pre & Post & $t$-test & $p$-value \\
\hline \multicolumn{5}{|c|}{ Study group } \\
\hline BI & $43.53 \pm 8.76$ & $34.5 \pm 8.76$ & 5.397 & $0.01 *$ \\
\hline BMI & $34.6 \pm 3.76$ & $32.5 \pm 3.34$ & 6.129 & $0.01 *$ \\
\hline $\mathrm{BF}(\%)$ & $42.9 \pm 6.81$ & $38.4 \pm 4.23$ & 4.265 & $0.01^{*}$ \\
\hline $\mathrm{WC}(\mathrm{cm})$ & $100.07 \pm 7.29$ & $92.0 \pm 9.99$ & 6.12 & $0.001 *$ \\
\hline $\mathrm{HC}(\mathrm{cm})$ & $119.73 \pm 9.33$ & $114.2 \pm 8.69$ & 4.50 & $0.001 *$ \\
\hline MM (\%) & $27.0 \pm 4.01$ & $29.9 \pm 2.90$ & -4.64 & $0.01 *$ \\
\hline WHR & $0.837 \pm 0.058$ & $0.808 \pm 0.088$ & 2.560 & $0.002 *$ \\
\hline \multicolumn{5}{|c|}{ Control group } \\
\hline BI & $41.3 \pm 9.04$ & $38.7 \pm 10.01$ & 2.722 & $0.02 *$ \\
\hline BMI & $33.2 \pm 3.11$ & $32.8 \pm 3.02$ & 2.638 & $0.02 *$ \\
\hline $\mathrm{BF}(\%)$ & $46.4 \pm 5.81$ & $44.8 \pm 5.45$ & 4.491 & $0.00 *$ \\
\hline $\mathrm{WC}(\mathrm{cm})$ & $24.1 \pm 2.92$ & $25.1 \pm 2.69$ & 1.52 & 0.96 \\
\hline $\mathrm{HC}(\mathrm{cm})$ & $114.3 \pm 7.67$ & $112.3 \pm 7.47$ & 0.96 & 0.09 \\
\hline $\mathrm{MM}(\%)$ & $24.10 \pm 2.92$ & $25.10 \pm 2.69$ & -4.81 & $0.01 *$ \\
\hline WHR & $0.87 \pm 0.805$ & $0.868 \pm 0.819$ & 0.349 & 0.73 \\
\hline
\end{tabular}

$\mathrm{BI}$ - body image; BMI - bdy mass index; $\mathrm{BF}$ - body fat; MM - muscle mass; WC - waist circumference; HC - hip circumference; WHR - waist-hip ratio; * - statistical significant difference

\section{Discussion}

Results from this study revealed a significant difference in the mean score of body image within the study and control groups post-intervention. However, there was an improvement in the body image between the study and control groups but it did not attain a significance level. This finding is in agreement with the result of a similar study which found out that college females who exercised for maintenance of a healthy weight or enhanced tone had lower body satisfaction and that those that exercised for the purposes of fitness and health had enhanced body satisfaction [22]. On the contrary, another study showed that participation in 6-week strength CET enhanced the body image of Slovakian primary school females [23]. Also, it was revealed that improvement in various dimensions of body image can be achieved by a relatively short duration circuit-weight training of college males and females in USA [24]. However, it should be noted that the six-week intervention 
utilized in this study improved the perception of body image but the observed improvement was not statistically significant between the groups. This may be attributed to the fact that the previous studies did not specifically target obese subjects. In addition, some of these studies involved only female subjects. Also, the age difference between the subjects of this study compared to previous studies may have contributed to the disparity in the results. The short duration of the present study may not have provided adequate time for a significant effect to be observed, considering the fact that the control group also received dietary counseling which may also have had an effect on the body image. The positive effect of exercise on the perception of body image maybe due to improvement or raised confidence in self-efficacy, improved fitness level and enhanced awareness of physical function. Dietary counseling, as well as exercise, resulted in obvious changes in both BMI and BI. People tend to feel better about their body when they observe positive changes in their body composition. This may explain the non statistical significance in BI when compared between the groups.

The present study reported a mean significant difference in the score of the cardiorespiratory fitness/aerobic capacity $\left(\mathrm{VO}_{2} \mathrm{max}\right)$ between the study and control group post-intervention. This corroborates with the results of a meta-analysis which demonstrated that resistance CET had an overall significant and large effect on $\mathrm{VO}_{2} \max$ (average of 9.7\%) in different populations [25]. Thus, the result of the present study has proven that CET can be a relevant intervention that improves the cardiorespiratory fitness and health status of obese individuals. It appears that adequate training intensity or stimulus and a short rest interval seem to be keys to eliciting changes in aerobic capacity.

The mean significant difference in heart rate recorded between the groups post-intervention is in consonance with the results of Mogharnasi et al. [26] which reported that heart rate had a significant change compared with the control group after 8 weeks of CET among obese male students. Also, results by Miller et al. [27] showed that a high-intensity circuit-training in sedentary obese men revealed a significant improvement in resting HR (16\% decrease). Therefore, it is most likely that 6 weeks duration of exercise in this study, was of the appropriate intensity to cause a significant change in resting heart rate. This can buttress the marginal effect of exercise intensity and duration on the resting heart rate. The ability of the heart rate to recovery after exercise depends on the extent to which the cardiovascular system functions to reverse the autonomic nervous system stimulants and adjusts the pressure receptors that are involved during exercise. Heart rate initial decrease was due to the reactivation attempts of parasympathetic nerves, with the slowed decrease due to continued activity of parasympathetic nerves and decreased impact of sympathetic nerves [28].

There was also a significant difference in the mean blood pressure (SBP and DBP) between the study and control group post-intervention. This is in consonance with Allyson et al. [29] who reported a significant reduction in central blood pressure, following a 4-week circuit CET intervention. In their study, it was asserted that though the mechanism was not examined, reduced central blood pressure may be associated with a decreased vascular resistance relating to the enhanced endothelial function (flow-mediated dilatation) and structure (carotid artery intima-media thickness). The reduction in blood pressure in this present study may be further explained by the postulation which states that a relationship exists between blood pressure and body composition; and that increased blood pressure is often associated with excess body fat, as such exercise for weight loss is often recommended as a strategy for decreasing blood pressure [30]. The Lower blood pressure associated with the study group is believed to be caused by a continuous reduction in systemic vascular resistance. Contributing to this effect is the signaling of the production and release of nitric oxide and prostacyclin from the vascular endothelium activated by the action of accumulated metabolites from skeletal muscle during exercise, which encourages enhanced vasodilation through relaxation of vascular smooth muscle [31].

The results of this study indicated that CET significantly decreased the percentage of body fat following the 6 weeks intervention. Previous studies $[32,33]$. also reported the same findings in their 12 weeks CET among obese individuals. Hence, these findings indicate that CET improves body fat in the obese population. This study showed a significant difference in the mean muscle mass between the study and control group post-intervention. This was supported by the findings of a similar study which indicated that exercise could significantly increase the lean body mass of obese people [34]. The study of Miller et al.[27] also proved that short-term high-intensity circuit 
training produced a significant improvement ( $2 \%$ increase) in lean muscle tissue percentage. Contrary to these findings, Kim et al. [32] reported that there was no significant difference in muscle mass between the groups post-intervention. They implied that the difference in the outcome of their study is likely to be a result of different types of exercise, time, duration, and intensity utilized.

The findings of this study also showed no significant difference in the mean BMI between the study and control group. This is in agreement with the result of a similar study that utilized a 10 week CET with no significant difference in body weight and invariably, BMI [35]. They suggested it was likely due to the relatively low loads of resistance exercise used in the study. Several research works have reported contradictory findings. A meta-analysis revealed that CET is effective in reducing body weight and BMI in adults and particularly in those with overweight and obesity [36]. The meta-analysis reported that the extent of weight reduction had a tendency to increase with an increase in the average BMI of the participants. It is imperative to recognize that these studies did not place the participants in their control group on dietary counseling. The short duration of the present study may not have provided adequate time for a significant effect to be observed, considering the fact that the control group was also placed on a dietary counseling which may also have an effect on the BW and invariably on the BMI. However, the present study did not measure calorie intake of the subjects. The result of this study should be interpreted with caution because this study has a limitation for not monitoring the dietary progression (calorie restriction) of the subjects.

The result of this study indicated no significant difference in the mean waist-hip ratio between the study and control group post-intervention. This is in agreement with the results of Ferreira et al. [37] who reported no significant changes in waist-hip ratio and waist circumference in 14 sedentary females within the age range of 33-45 years, who participated in a 10-week resistant CET. The insignificant difference observed in the variables was in line with the literature that body composition changes take place slowly in human exercise studies and the magnitude of the change is small [36]. However, these results are at variance with the findings of Billy et al. [39] who observed a reduction in the waist-hip ratio of participants after high-intensity CET. The difference observed in the study may be due to the different modes of training, duration, intensity, and types of exercises adopted. However, the findings of this study may be a result of the dietary counseling which control group was placed on. Consequently, it could be assumed that both CET and dietary counseling improves fat loss, and as such the difference in fat loss between the groups as a result of 6-week CET may not be significant. Moreover, further research for a longer period of time should be carried out to determine the combined effect of CET and and only dietary counseling on WHR of obese individuals. More so, calculation of calorie intake/loss and its monitoring will authenticate the effectiveness of dietary counseling as an intervention for fat loss among obese individuals.

More importantly, it is worthy of note that all the body composition parameters including visceral fat, recorded a significant change post-intervention in the study group. This has farreaching health implications in the obese population. Chronic inflammation has a strong positive correlation with overweight and obesity [40]. Considering the implication of inflammation in cardiovascular disease, one of the strategies to reduce the disease is to improve body composition and inflammatory markers by adequate participation in exercise (comprising of aerobic and resistance exercises). Also, excess body fat accumulation, in particular visceral fat, is associated with insulin resistance and the occurrence of type 2 diabetes (T2D) [41]. However, it has also been shown that adequate intensity and volume of moderate-intensity results in a decline in body fat and visceral fat and consequently reducing the risk of occurrence or incidence of T2D [41].

\section{Conclusion}

A 6-week circuit exercise intervention has proven to be an efficient intervention for the improvement of body image, cardiorespiratory parameters, and body composition of obese population, specifically young adults. Participation in circuit exercise should be encouraged amongst obese individuals as they are more at risk of developing cardiovascular diseases and prone to battling with body size dissatisfaction. In addition, circuit exercise can be self administered and as such, the economic/financial burden (in a poor developing nation) of registering in a regular gym 
setting for weight reduction is reduced. Physiotherapists should be involved in the multidisciplinary team management of obesity.

\section{Disclosure statement}

No author has any financial interest or received any financial benefit from this research.

\section{Conflict of interest}

The authors state no conflict of interest.

\section{References}

1. Al-Sendi AM, Shetty P, Musaiger AO, Myatt M. Relationship between body composition and blood pressure in Bahraini adolescents. $\mathrm{Br} J$ Nutr. 2003;90(4):837-844; doi: 10.1079/bjn2003963.

2. Ali SM, and Lindstrom M. Socioeconomic, psychosocial, behavioral and psychological determinants of BMI among young women: differing patterns for underweight and overweight/obesity. Eur J Public Health. 2006;16(3):324-330; doi: 10.1093/eurpub/cki187.

3. Allyson KG, Tia RW, Lauren NC, Cassndra CD, Kelly CM, Avery NP et al. Effects of circuit exercise training on vascular health and blood pressure. Prev Med Rep. 2018;10:106-112; doi: 10.1016/j.pmedr.2018.02.010.

4. Bastug G, Goral K, Ekici SH, Karatan OV. Investigation of Cardio Exercise's Effects on Body Perception and Life Satisfaction in Relation with Some Parameters. Turk J Sport Exerc. 2018;20:57-62; doi:10.15314/tsed.427664.

5. Sperlich B, Wallmann-Sperlich B, Zinner C, Von Stauffenberg V, Losert H and Holmberg H-C (2017) Functional High-Intensity Circuit Training Improves Body Composition, Peak Oxygen Uptake, Strength, and Alters Certain Dimensions of Quality of Life in Overweight Women. Front Physiol. 2017;8:172. doi: 10.3389/fphys.2017.00172

6. Boyland EJ and Whalen R. Food advertising to children and its effects on diet: review of recent prevalence and impact data. Pediatr Diabetes. 2015;16(5):331-337; doi: 10.1111/pedi.12278

7. Donnelly JB, Jakicic JM, Manore MM, Rankin JW, Smith BK. Appropriate physical activity intervention strategies for weight loss and prevention of weight regain for adults. Med Sci Sports Exerc. 2009;41(2):459-71; doi: 10.1249/MSS.0b013e3181949333.

8. Erem C. Prevalen+ce of Overweight and Obesity in Turkey. IJC Metabolic and Endocrine 2015; 8:38-41; doi.org/10.1016/j.ijcme.2015.07.002

9. Ferreira FC, Medeiros AI, Nicioli C, Nunes ED, Shiguemoto GE, Prestes J et al. Circuit Resistance Training in Sedentary Women: Body Composition and Serum Cytokine Levels. Appl Physiol Nutr Metab. 2010;35(2):163-171; doi: 10.1139/H09-136

10. Francisco AM, Jacobo AR, Domingo JR, Pedro EA. Effectiveness of Resistance Circuit- Based Training for Maximum Oxygen Uptake and Upper-Body One-Repetition Maximum Improvements: A Systematic Review and Meta-Analysis. Sports Med 2017;47(12):2553-2568; doi: 10.1007/s40279-017-0773-4

11. Fujitsuka S, Koike Y, Isozaki A, Nomura Y. Effect of 12 weeks of strenuous physical training on hematological changes. Military Med. 2005;170(7):590-594; doi: 10.7205/milmed.170.7.590.

12. Furnham A, Badmin N, Sneade I. Body image dissatisfaction: Gender differences in eating attitudes, self-esteem, and reasons for exercise. J Psychol. 2002;136(6):581-596; doi:10.1080/00223980209604820

13. Goedecke JH, Micklesfield LK. The effect of exercise on obesity, body fat distribution and risk for type 2 diabetes. Med Sport Sci. 2014;60:82-93; doi: 10.1159/000357338

14. Greenberg AS and Obin MS. Obesity and the role of adipose tissue in inflammation and metabolism. Am J Clin Nutr. 2006;83(2):461-465; doi: 10.1093/ajcn/83.2.461S.

15. Harber MP, Fry AC, Rubin MR, Smith JC,Weiss LW. Skeletal muscle and hormonal adaptations to circuit weight training in untrained men. Scand J Med Sci Sports. 2004;14(3):176-185; doi: 10.1111/j.1600-0838.2003.371.x.

16. Haskell WL, Lee IM, Pate RR, Powell KE, Blair SN, Franklin BA et al. Physical activity and public health: Updated recommendation for adults from the American College of Sports 
Medicine and the American Heart Association. Med Sci Sports Exerc. 2007;39(8):1423-1434; doi: 10.1249/mss.0b013e3180616b27.

17. Henry RN, Anshel MH, Michael Timothy. Effects of Aerobic and Circuit Training on Fitness and Body Image Among Women. J Sport Behav. 2006;29:281-292.

18. International Society for the Advancement of Kinathropometry. International standards for Anthropometric Assessment 2001. Available @ www. Isakonline.com

19. Uth N, Sørensen H, Overgaard K, Pedersen PK. Estimation of VO2max from the ratio between HRmax and HRrest--the Heart Rate Ratio Method. Eur J Appl Physiol. 2004;91(1):111-115;. doi: 10.1007/s00421-003-0988-y

20. Jaffrin MY. Body composition determination by impedance. Curr opin Clin nutr Metab Care. 2009;12(5):482-486; doi: 10.1097/MCO.0b013e32832da22c

21. Ignaszewski $\mathrm{M}$, Lau $\mathrm{B}$, Wong $\mathrm{S}$, Isserow $\mathrm{S}$.The science of exercise prescription: Martti karvonen and his contributions. BCMJ. 2017;59(1):38-41

22. Kim JW, Ko YC, Seo TB, Kim YP. Effect of circuit training on body composition, physicalfitness, and metabolic syndrome risk factors in obese female college students. J Exerc Rehabil. 2018;14(3):460-465; doi: 10.12965/jer.1836194.097.

23. Kostrzewa-Nowak D, Nowak R, Jastrzębski Z, Zarebska A, Bichowska M, DrobnikKozakiewicz I et al. Effect of 12- week-long aerobic training programme on body composition, aerobic capacity, complete blood count and blood lipid profile among young woman. Biochem Med (Zagreb). 2015;25(1):103-113; doi: 10.11613/BM.2015.013.

24. Kyle TK, Dhurandhar EJ, Allison DB. Regarding Obesity as a Disease: Evolving Policies and Their Implications. Endocrinol Metab Clin North Am. 2016;45(3):511-520; doi: 10.1016/j.ecl.2016.04.004.

25. Leslie HW, Cris AS, Lori AB, Shields AT, Lucy WP, Connie WB. Effects of aerobic and/or resistance training on body mass and fat mass in overweight or obese adults. J Appl Physiol. 2012;113(12):1831-1837; doi: 10.1152/japplphysiol.01370.2011

26. Liao C, Tsauo1 J, Huang S, Ku J, Hsiao D, Liou T. Effects of elastic band exercise on lean mass and physical capacity in older women with sarcopenic obesity: A randomized controlled trial. Sci Rep. 2018;8(1):2317-2314; doi: 10.1038/s41598-018-20677-7

27. Lucia P, Janka P, Petra P, Klaudia K, Olympia M. The effects of a 6-week strength and endurance circuit training intervention on body image in Slovak primary school girls. J Phys Edu Sports. 2018;18 Suppl 1,64:459-464; doi:10.7752/jpes.2018.s164

28. Miller MB, Pearcey G E, Cahill F, McCarthy H, Stratton SB., Noftall JC et al. The effect of a short- term high-intensity circuit training program on work capacity, body composition, and blood profiles in sedentary obese men: a pilot study. BioMed Res Int. 2014;2014: 1155-1165; doi: $10.1155 / 2014 / 191797$

29. Mogharnasi M, Eslami R, Behnam B. Effects of Endurance and Circuit Resistance Trainings on Lipid Profile, Heart Rate, and Hematological Parameters in Obese Male Students. Ann Appl Sport Sci. 2014;2(4):11-22; doi: 10.18869/acadpub.aassjournal.2.4.11

30. Niebauer J, Cooke JP. Cardiovascular effects of exercise: role of endothelial shear stress. J Am Coll Cardiol. 1996;28(7):1652-1660; doi.org/10.1016/S0735-1097(96)00393-2

31. Pedišić Ţ, Jurakić D, Rakovac M, Hodak D, Dizdar D. Reliability of the Croatian long version of the international physical activity questionnaire. Kinesiol. 2011;43(2):185-191; UDC 303.6:796.012.1(497.5)

32. Regaieg S, Charfi N, Abid M. The effects of an exercise training program on body composition and aerobic capacity parameters in Tunisian obese children. Indian $\mathbf{J}$ Endocrinol Metab 2013;17(6):1040-1045; doi: 10.4103/2230-8210.122619

33. Sarría A, García-Llop L, Moreno L, Fleta J, Morellón M, Bueno M. Skinfold thickness measurements are better predictors of body fat percentage than body mass index in male Spanish children and adolescents. Eur J Clin Nutr. 1998;52(8):573-576; doi: $10.1038 /$ sj.ejcn.1600606.

34. Senekal M and Kunneke E. The association between the body mass index of first year female university students and their weight-related perceptions and practices, psychological health, physical activity and other physical health indicators. Pub Health Nutr. 2006;9(2):234-43; doi: 10.1079/phn2005846. 
35. Seo YG, Noh HM, Kim SY. Weight loss effects of circuit training interventions: A systematic review and meta- analysis. Obes Rev. 2019;20(11):1642-1650; doi: 10.1111/obr.12911.

36. Seol-Jung K, Jong-Hyu K, Zhao G, Young-Sook Y, Jea-Ryang Y, GIi-Chul H et al. Effects of 12-week circuit exercise program on obesity index, appetite regulating hormones, and insulin resistance in middle-aged obese females. J Phys Ther Sci. 2018;30(1):169-173; doi: 10.1589/jpts.30.169

37. Veale D, Eshkevari E, Kanakam N, Ellison N, Costa A, Werner T. The Appearance Anxiety Inventory: Validation of a Process Measure in the Treatment of Body Dysmorphic Disorder. Behav Cogn Psychother. 2014;42(5):605-616; doi: 10.1017/S1352465813000556.

38. Warburton DE, Gledhill N, Quinney A. Musculoskeletal Fitness and Health. Canad J Appl Physiol. 2001;26(2):217-237; doi: 10.1139/h01-013.

39. Warburton DE, Nicol CW, Bredin SS. Health benefits of physical activity: the evidence. CMAJ. 2006;174(6):801-809; doi: 10.1503/cmaj.051351.

40. Westerterp KR. Exercise, energy balance and body composition. Eur J Clin Nutr. 2018;72:1246-1250; doi.org/10.1038/s41430-018-0180-4

41. World Health Organization. Obesity. 2015 https://www.who.int/topics/obesity/en/ 